- This paper describes the case management of several cases of patients with confirmed CJD, suspected CJD or 'at-risk' from CJD.

- Background infection control information relevant to CJD is provided.

- Practical advice is given on the clinical management of patients with CJD.

\title{
Prion diseases and dental treatment: principles and practice of patients with/suspected or at-risk of CJD: Case reports
}

\author{
A. J. Smith, ${ }^{1}$ M. P. Sweeney ${ }^{2}$ and J Bagg ${ }^{3}$
}

The emergence of variant CJD (VCJD) in the last decade has heightened awareness of the need for rigorous infection control precautions in all healthcare environments. It has also raised particular problems in relation to the clinical management of patients who are either suffering from prion diseases or who are perceived as being 'at risk'. From the healthcare perspective CJD is of concern because at present it is an incurable, fatal disease and the causative agent, abnormal prion protein, is resistant to conventional inactivation procedures. Many healthcare workers are concerned about the risks of cross-infection when treating such patients. There are a number of guidelines for the management of CJD patients, though the information they provide sometimes appears contradictory and may require some interpretation in the clinical setting. This paper is based on real-life case scenarios and the advice given to dentists for the management of a group of patients with different manifestations of prion diseases.

\section{INTRODUCTION}

In 2000, the Department of Health established the CJD advice network. This group comprises individuals from a wide range of healthcare professions and volunteer workers with experience in the management and treatment of patients with CJD. The aim of the network is to share the experiences of the management of patients with CJD with other healthcare professionals. There are a number of dentally qualified individuals on the network to provide support and advice to the dental profession on the management of patients with CJD. Since the network was established there have been over 20 cases where advice has been sought on the dental management of patients with CJD, those with suspected CJD or those 'at-risk' of CJD.

\footnotetext{
${ }^{1}$ Senior Lecturer in Microbiology, ${ }^{2}$ Senior Lecturer in Special Needs Dentistry, ${ }^{3}$ Professor of Clinical Microbiology, Infection Research Group, Glasgow Dental Hospital and School, 378 Sauchiehall Street, Glasgow G2 3JZ

Correspondence to: A. Smith,

E-mail:a.smith@dental.gla.ac.uk
}

\section{Refereed Paper}

Received 19.03.03; Accepted 10.07.03

doi:10.1038/sj.bdj.4810542

( ) British Dental Journal 2003; 195: 319-321
Prion diseases may present as infectious, genetic or sporadic forms of the disease (Table 1). It is important to have a confirmed diagnosis of the type of CJD from which the patient is suffering, since this may affect future treatment of family members of the case. Prion diseases are invariably fatal, frequently with a rapid decline in health, particularly in the latter stages of the disease. However, the main challenge from the infection control perspective is that the agents primarily responsible for CJD, abnormal prions, are remarkably resistant to conventional sterilization processes. Current evidence suggests that normal social or routine clinical contact does not present a risk to healthcare workers, families or others. There is no evidence of infectivity in saliva ${ }^{1-3}$ and there is uncer-

\begin{tabular}{ll}
\hline \multicolumn{2}{l}{ Table 1 Different types of Human CJD } \\
\hline Mode of acquisition of CJD & Type of CJD \\
\hline Sporadic & Sporadic CJD \\
Inherited & Familial CJD \\
Acquired - Human & Kuru, iatrogenic CJD \\
Acquired - Bovine & Variant CJD
\end{tabular}

tainty about the potential for transmission from blood, but universal infection control precautions should minimise any risk that may exist.

There are a number of guidelines ${ }^{3-5}$ for the management of patients with confirmed CJD, those suspected of suffering from CJD and those 'at-risk' of CJD. The definition of the 'at-risk' group is shown in Table 2.

The aim of this paper is to describe the infection control protocols recommended for actual dental treatment in a range of patients in these groups.

\section{Table 2 Definition of 'at-risk' patients}

Definition of 'at-risk' = Asymptomatic but potentially at-risk of developing disease: -recipients of hormone derived from human pituitary glands eg growth hormone, gonadotrophin. -recipients of human dura mater grafts -people with a close (blood line) family history of CJD.

Source: Advisory Committee on Dangerous Pathogens and Spongiform Encephalopathy Advisory Committee (1998). Transmissible Spongiform Encephalopathy agents: Safe working and the prevention of infection. London, Stationery Office. 


\section{SCENARIO 1 - A PROBABLE CASE OF VCJD} Patient A had been recently diagnosed with probable vCJD. The unit caring for the patient wished to formulate a protocol for dental treatment of the patient should it become necessary. Unfortunately the patient died prior to the provision of any dental treatment. However, pre-planning is essential and the recommendations which were given for treatment were as follows.

\section{Case management}

It is unlikely that general dental practitioners will be directly involved in the provision of care for patients suffering, or suspected to be suffering, from any form of CJD. Such patients may be exhibiting early neurological signs and symptoms of disease and their care will be mostly based in a hospice or hospital. Appropriate treatment planning for this group of patients is best performed by dentists with experience of special care dentistry.

Because of the rarity and special features of the disease, local care services, including dental professionals, may need specialist support and advice. The main coordinating source of advice is the National CJD Surveillance Unit (NCJDSU). The NCJDSU based at the Western General Hospital, Edinburgh, has a national care coordinator to provide specialist expertise in CJD and to act as an information resource for carers and professionals.

All patients with a clinical diagnosis of CJD should have a named key worker identified to co-ordinate care for patient and family, regardless of the setting in which care is given. It is vital that appropriate members of the dental team are involved at an early stage to provide advice and, if necessary, treatment for the patient. Key workers should be aware of the importance of preventive dental advice as part of the overall care of such patients. All healthcare professionals should be alert to the rapidity of deterioration that occurs in CJD and reassess patients' needs frequently. Of note for dentists is the presence of neurological deficits, such as sensory and motor impairments, which may manifest in the head and neck region. Such features may present such as atypical facial pain.

Treatment guidelines have been suggested $^{6}$ for the management of confirmed and suspected CJD cases. There is one major difference from normal universal infection control procedures, namely the requirement for all instruments, including handpieces, to be single-use in those cases where the diagnosis is confirmed. This may entail a period of quarantining instruments whilst the diagnosis is being made. In all other respects, the management of confirmed or suspected cases of CJD for routine dental procedures requires no special infection control precautions for operator or patient above the universal infection control precautions adopted for all patients. Some authors have suggested additional precautions for this group of patients, such as a separate water supply for handpieces and suction systems. ${ }^{7}$ However, this has not been universally accepted, ${ }^{8}$ since there is no evidence to suggest that prions are transmitted via this route. Clearly, protocols for the disposal of non single-use devices such as handpieces must be considered before treatment, in conjunction with the local infection control team. Forward planning, both logistical and financial, are essential.

\section{SCENARIO 2 - A RELATIVE OF A PATIENT WITH VCJD}

Patient B attended his / her regular GDP with a complaint of toothache. During the course of the examination the patient confirmed that a close family relative had died from vCJD. The GDP performed emergency dental treatment to relieve the dental source of the pain. At the conclusion of the visit the GDP placed all the re-usable dental equipment in a secure box and instructed the patient to take the instruments home in the event that further dental treatment may be required.

\section{Case management}

Guidance on the management of family members of patients who are suffering or have died from VCJD has, in the past, been confusing. As a result, some family members have been refused treatment by their general dental practitioner, community dental services and hospital dental units. Recent advice has clarified that family members of $\mathrm{VCJD}$ cases do not require any additional infection control precautions. ${ }^{5}$

Following discussion with the general dental practitioner of patient $\mathrm{B}$, the instruments used to treat the patient were returned to the practice and reprocessed according to the normal routine. The patient continues to attend the general dental practitioner for routine dental treatment with no special infection control precautions.

\section{SCENARIO 3 - AN ELDERLY NURSING HOME RESIDENT WITH SUSPECTED CJD}

A community dental officer was asked to review Patient C, an elderly individual with a suspected diagnosis of sporadic CJD, who had a broken denture. Following examination of the patient a decision was made to repair the denture, but advice was sought on suitable infection control measures.

\section{Case management}

There is no evidence of infectivity in saliva for either human or animal prion diseases. ${ }^{1-3}$ Adherence to universal infection control precautions is sufficient to minimise the risks from abnormal prions or any other infectious disease likely to be encountered in this environment. The infection control advice for the replacement of patient C's denture followed routine protocols of impression taking followed by a thorough rinsing of the impression under running tap water and soaking for 5 minutes in a freshly prepared solution of a proprietary dental impression disinfectant. The denture laboratory was informed and reassured. The denture was repaired and returned to the patient.

\section{SCENARIO 4 - A PATIENT 'AT-RISK' OF CJD}

Following a routine medical history check, patient D revealed that he / she had received artificial growth hormone as a child during the 1970s. This information would classify the patient as 'at risk' for iatrogenic CJD. No urgent dental treatment was required and the general dental practitioner sought advice on infection control precautions for this patient.

\section{Case management}

The management of patients in the 'at-risk' group is controversial, with a number of guidelines providing advice. ${ }^{3-5}$ Some of the guidelines recommend stringent instrument decontamination procedures, ${ }^{3}$ which are difficult to implement in most general dental practices. In this particular case, reference was made to the World Health Organisation (WHO) guidelines ${ }^{4}$ on the management of patients 'at-risk' of CJD.

In summary, the decision was made that for routine dental procedures, which the WHO guidelines currently regard as low risk in relation to potential transmission of abnormal prion protein, no special infection control procedures were necessary for the treatment of this patient. The re-processing of re-usable dental instruments, such as, high speed handpieces and hand instruments, was performed according to the usual surgery protocol of cleaning and sterilising in a bench top autoclave at the standard time and temperature settings. In addition, advice was given to the general dental practitioner that reprocessing of endodontic files was not recommended and that these instruments should be for single patient use only. In the light of current evidence on the difficulty of cleaning endodontic instruments, ${ }^{9}$ it is the authors' view that they should be viewed as single use for all patients, regardless of their medical history.

If the WHO guidelines ${ }^{4}$ are accepted and followed for the management of 'at 
risk' patients, then it removes the need to seek information in the medical history relating to previous injections of pituitary hormones, placement of dura mater grafts and family history of CJD. This is the current policy at Glasgow Dental Hospital and at the practice providing care for patient D. However, a high standard of infection control must be applied for all patients, with particular emphasis on cleaning of reusable devices prior to sterilisation and care to ensure that no single-use items are re-processed.

\section{DISCUSSION}

The emergence of CJD in the last decade has heightened awareness of the need for thorough infection control procedures, not just for confirmed or suspected cases of CJD but for all patients in a number of healthcare environments. The current advice from the Department of Health following a risk assessment of the risks of transmission of vCJD via surgical instruments is that the effectiveness of routine instrument decontamination should be of the highest standard to minimise the risks of onward transmission. ${ }^{10}$ Whilst current data suggest that prion proteins cannot be detected in pulpal tissue from patients with sporadic $\mathrm{CJD},{ }^{11}$ it is unknown whether the agent of vCJD is present in dental pulps. There is an additional complication because the agent of VCJD is also present in lymphoid tissue, ${ }^{12}$ with possible implications for transmission during certain oral and dental procedures. There is animal model work that suggests the potential for onward transmission of scrapie agent via the dental route in a hamster model, ${ }^{13}$ though direct extrapolation to human forms of CJD is difficult, due to differences in the strain of prion protein used and the experimental host.

There are a number of guidelines available from national and international advisory bodies which provide advice on the management of patients affected by CJD. These guidelines have been compiled in the face of relatively little direct clinical, epidemiological or experimental evidence, especially in relation to vCJD. This can lead to difficulties in establishing treatment protocols for affected patients, with many healthcare workers delaying or refusing to provide treatment because of fears over cross-infection. The case studies provided in this paper present practical and pragmatic advice that was used for the management of four cases, to highlight the range of presentations of patients presenting with prion diseases. However, this is a rapidly moving area and these case studies do not provide the definitive answer to case management, which may be modified in the light of new information. The cases do, however, illustrate that up to date advice is available from a number of sources, a selection of which is provided below. In addition, practitioners should be aware that they can liase with their local infection control teams to assess the risks from each case and devise local protocols in the light of current guidelines for the management of patients with CJD. Patients with prion diseases, together with their families, have overwhelming medical, social and psychological problems. Dental health problems only add to that burden and the dental profession can play a very important role in alleviating such difficulties when they arise.

1. Hadlow W J, Kennedy R C, Race R E. Natural infection of Suffolk sheep with scrapie virus. J Infect Dis 1982: 146: 657-664.

2. Brown P, Gibbs C J Jr, Rodgers-Johnson P, et al. Human spongiform encephalopathy: the National Institute of Health series of 300 cases of experimentally transmitted disease. Ann Neurol 1994; 35: 513-29.

3. Advisory Committee on Dangerous Pathogens and Spongiform Encephalopathy Advisory Committee (1998). Transmissible Spongiform Encephalopathy agents:Safe working and the prevention of infection. London, Stationery Office.

4. WHO/CDS/APH/2000.3 WHO infection control guidelines for transmissible spongiform encephalopathies.

(www.who.int/csr/resources/publications/bse/WHO_ CDS_CSR_APH_2000_3/en/)

5. Department of Health (2000): Creutzfeldt-Jacob disease: Guidance for healthcare workers. (www.doh.gov.uk/cjd/healthworkers.htm)

6. Smith A J, Sweeney M P. Oral Health for patients with CJD. Briefing sheet 7 .

(http://www.hbsef.org/brief7andrewsmithdentist.htm)

7. Porter S, Scully, C, Ridgway G L, Bell J. The human transmissible spongiform encephalopathies (TSEs): implications for dental practitioners. Br Dent J 2000; 188: 432-436.

8. Smith A J, Martin M V. Managing patients with TSE's.
BrDent J2000; 189: 62.

9. Smith A J, Bagg J, Aitken J, Dickson M. Contaminated dental instruments. J Hosp Infect 2002; 51: 233-235.

10. Department of Health. Risk assessment for transmission of VCJD via surgical instruments: $A$ modelling approach and numerical scenarios (2001) (www.doh.gov.uk/cjd/riskassessmentsi.htm).

11. Blanquet-Grossard F, Sazdovitch V, Jean A, et al. Prion protein is not detectable in dental pulp from patients with Creutzfeldt-Jakob disease. J Dent Res 2000; 79: 700.

12. Hill A F, Butterworth R J, Joiner S, et al. Investigation of variant Creutzfeldt-Jakob disease and other human prion diseases with tonsil biopsy samples. Lancet 1999; 353: 183-189.

13. Ingrosso L, Pisani F, Pocchiari M. Transmission of the 263K scrapie strain by the dental route. J Gen Virol 1999; 80: 3043-3047.

\author{
Sources of further advice \\ 1. National Care Co-ordinator for CJD \\ Gordon McLean \\ National CJD Surveillance Unit, \\ Western General Hospital, \\ Crewe Road, \\ Edinburgh. \\ Tel: 01315372129 \\ www.cjd.ed.ac.uk

\section{CJD Support Network} \\ Gillian Turner \\ National CJD Co-ordinator \\ CJD Support Network \\ Birchwood \\ Heath Top \\ Ashley Heath \\ Market Drayton, \\ Shropshire \\ Tel:01630 673993 \\ www.cjdsupport.net \\ email:info@cjdsupport.net
}

\section{Department of Health CJD advice network} A virtual network of a wide range of healthcare workers who have had experience of treating patients with CJD. They can be contacted via the CJD support network. The network includes two dentists, one of whom is a general dental practitioner.

\section{The Prion Unit}

Prion Unit,

Dept of Neurology,

St Mary's hospital,

Praed St,

London

Tel: 02078866883

The Prion Unit at St Mary's Hospital, London. Provides assessment, diagnosis and on-going support for patients suffering from CJD.

\section{The Human BSE Foundation}

\section{Tel 0191389415}

www.hbsef.org

A voluntary organisation run by families of VCJD

patients aimed at helping relatives, friends and carers of $v C J D$ patients.

\begin{abstract}
Addendum
Following submission of this publication the Department of Health has recently published updated guidance for healthcare workers on safe working with transmissible spongiform encephalopathies (TSEs) such as Creutzfeldt-Jakob Disease (CJD). This guidance replaces the edition issued in March 1998 and are published on-line at www.doh.gov.uk/cjd/tseguidance.

The revised guidelines recognise the low risk of transmission of infection from denta instruments, provided that optimal standards of infection control and decontamination are maintained. In practice this means that instruments used on all patients in the 'at-
\end{abstract}

risk' category can be treated in the same manner as those for all other patients. This recommendation is also extended to routine dentistry in confirmed or suspected CJD cases, negating the requirement to destroy or quarantine instruments. These guidelines re-inforce the notion that any patient or their relatives may be treated in general dental practice (in the same manner as any member of the general public). The logical extension of these guidelines removes the necessity to identify patients in the 'at-risk' category when taking a medical history prior to dental treatment. It will be clear to readers that these new guidelines would have simplified and expedited the management of the four patients described in this paper. 